\title{
Surgical Correction of Spontaneous Outflow Graft Twisting of HeartMate III via a Subcostal Approach
}

\author{
Hidefumi Nishida ${ }^{1}$, Valluvan Jeevanandam ${ }^{1}$, and Takeyoshi Ota ${ }^{1}$ \\ ${ }^{1}$ University of Chicago
}

August 5, 2020

\begin{abstract}
We experienced two cases of successful surgical correction of HeartMate 3 outflow graft twisting through a subcostal approach. They were diagnosed with computed tomography or pull back pressure measurement. Technically, a subcostal approach allowed us to access directly to the twisted outflow graft and the device connector in a less invasive fashion without a re-sternotomy. Diagnostic modality and surgical tips are presented.
\end{abstract}

\section{Introduction}

HeartMate 3 is a centrifugal-flow left ventricular assist device(LVAD) and the most updated version of circulatory support device. It has been reported that the device provides less incidences of device-related complications compared to the previous version of HeartMate ${ }^{1}$. However, there are a number of reports of late spontaneous outflow graft twisting events exclusively to HeartMate $3^{2,3}$.

\section{Case}

Case 1; A 54 year-old female was admitted due to shortness of breath and persistent LVAD low flow alarm. Her initial surgery(HeartMate 3 insertion with mitral and tricuspid valve repair) was 2 years and 6 months prior to this presentation. The LVAD pump flow was $1.4 \mathrm{~L} /$ minute with $5400 \mathrm{rpm}$ on admission, which was significantly lower than the baseline(i.e. $4.0 \mathrm{~L} /$ minute with $5400 \mathrm{rpm}$ ). An echocardiography revealed that the aortic valve was open with every beat with a dilated left ventricle. While lactate dehydrogenase(LDH) level was slightly elevated $(256 \mathrm{U} / \mathrm{l})$, serum haptoglobin $(145 \mathrm{mg} / \mathrm{dl})$ and indirect bilirubin $(0.2 \mathrm{mg} / \mathrm{dl})$ were within normal range. A computed tomography angiography(CTA) showed significant outflow graft narrowing at the vicinity of the LVAD pump(Figure 1A,1B).

Case 2; A 38 year-old male presented with shortness of breath, abdominal distension, nausea and vomiting. He had had the initial HeartMate 3 implantation and mitral valve repair 1 year and 6 months before. His pump flow was slightly lower than his baseline, specifically $4.0 \mathrm{~L} /$ minute with $6200 \mathrm{rpm}$ on admission and $5.1 \mathrm{~L} /$ minute with $6200 \mathrm{rpm}$ as baseline. An echocardiography demonstrated that the aortic valve was open with every beat. Lab data showed that lactate dehydrogenase(LDH) level was elevated(425U/1), serum haptoglobin was low $(36 \mathrm{mg} / \mathrm{dl})$ and indirect bilirubin was elevated $(3.1 \mathrm{mg} / \mathrm{dl})$. An outflow graft angiography was performed, which showed an apparent outflow graft twisting at the proximal portion of the outflow graft(Figure 1C) with a significant pressure gradient about $100 \mathrm{mmHg}$ at the twisting by a pull back pressure measurement(Figure 1D,1E).

Both cases were taken to the operating room with a diagnosis of outflow graft twisting/kinking. A subcostal approach was utilized to access directly to the proximal portion of the outflow graft. A reverse-J shape incision was made in the subcostal area and the outflow graft was exposed enough to access to the connection between the outflow graft and the device body. A Rultract retractor (Rultract Inc., Ohio) was used to lift 
the rib to obtain a reasonable exposure (Figure 2A). The bend relief was identified, the plastic frames of the bend relief were partially removed, and the bend relief was longitudinally opened to exposed the outflow graft. It was found that the outflow graft was twisted about 3-4 cm from the device connector (Figure 2B). While the bend relief was stuck to the surrounding tissue, the bend relief was detached from the connector using the HeartMate accessory instrument. Then, the connector was rotated in a direction for untwisting the outflow graft. A curved-up Rongeur forceps was helpful to rotate. In both cases, it required to rotate in a counter clockwise fashion in 120 degree to fix the twisting(Figure 2C,2D). Hemodynamics and LVAD parameters were immediately improved. We did not place an outflow graft clip given the risks to injure the surrounding structures during a dissection with a relatively limited exposure. The opening of the bend relief was covered with a Gore-Tex soft tissue patch(WL Gore \& Assoc Inc, Flagstaff, Ariz). After decent hemostasis, the incision was closed in layers in the routine fashion. The postoperative course was uneventful. No recurrent issue has been noted for 4 months of the follow up period.

\section{Discussion}

The first clinical trial for the HeartMate 3 was initiated in $2014 .{ }^{4}$, and it was approved by the Food and Drug Administration(FDA) for the commercial use in 2017. It was reported that the HeartMate 3 pump showed either comparable or more favorable outcomes compared to the HeartMate 2 in terms of device-related complications such as a remarkable reduction in stroke rates ${ }^{1}$. However, there has been a device-related complication exclusive to the HeartMate 3, which is spontaneous outflow graft twisting in a long-term phase. Although the mechanism of late graft twist has not been clearly understood, the helical heart motion, ventricular remodeling, and/or metallic swivel joint configuration have been considered to lead to this issue ${ }^{2,3}$. According to the report from MOMENTUM3 trial, the incidence of late outflow graft twisting was $1.6 \%$ and the median duration from the index surgery was 544 days $^{2}$. In our case series, the incidence was $2.4 \%(2 / 84)$ and its median duration from the index implantation was 495days. As for diagnostic modality, it is generally recommended to obtain a CTA to detect the outflow graft twisting. In our first case, a CTA revealed a narrowing of the outflow graft, and the final diagnosis of twisting was confirmed during surgery. Thrombus formation inside the outflow graft would be an important differential diagnosis because it would need a different surgical strategy to avoid embolic events. However, it is sometime difficult to differentiate those diagnoses only with a CTA although it is known that most of late outflow graft twisting occurred at a proximal portion of the graft near the device connection ${ }^{2,3}$. In our $2^{\text {nd }}$ case, we performed an outflow graft angiography and it was very helpful to confirm the outflow graft twisting preoperatively. The graft angiography clearly showed a graft twisting and the pressure measurement demonstrated significant pressure gradient at the twisted point. An outflow graft angiography would be recommended in patients with a suspicious outflow graft twisting.

In terms of surgical approaches, a full resternotomy is one of options. However, it would require a full dissection of the heart and the device to fix the problem, which might need a cardiopulmonary bypass. A left thoracotomy could be another option. It will provide a reasonable access to the device body, however it would suffer from a limited exposure to access the main target; specifically the outflow graft which lies down anterior/medial side of the chest. We utilized a subcostal approach which allow us to access directly to the target area(i.e. the outflow graft and the device connector) in a less invasive fashion, and we successfully fixed the problems.

As a number of this issue being reported, outflow graft connector clips to avoid a twisting waere released in October 15 ${ }^{\text {th }}, 2018$ by Abbott(Abbott Park, IL). Eventually, integrated pump cover was released on November $11^{\text {th }}, 2019$. Index implantations for both our cases were before the clip was released. It is important to note that we did not place a clip during the surgery due to anatomical reasons. We believe that the connector would not rotate again due to severe adhesion. However, this could be a trade-off in a subcostal approach and we would need a close follow up for our patients.

In conclusion, we have experienced two cases of spontaneous outflow graft twisting in patients supported with a HeartMate 3 , and successfully treated with a surgical repair using a subcostal approach. 
Acknowledgements: none

Disclosures: Dr Jeevanandam is a scientific advisor of Abbott

The institutional review board of our institution approved this study and waived the requirement for informed consent.

Author contributions;

Hidefumi Nishida; concept, drafting article

Valluvan Jeevanandam; Critical revision of article, Approval of article

Takeyoshi Ota; Critical revision of article, Approval of article

\section{References}

1. Mehra MR, Uriel N, Naka Y, et al. A Fully Magnetically Levitated Left Ventricular Assist Device Final Report. N Engl J Med. 2019 Apr 25;380(17):1618-1627

2. Mehra MR, Salerno C, Naka Y, et al. A tale of the twist in the outflow graft: An analysis from the MOMENTUM 3 trial. J Heart Lung Transplant. 2018 Nov;37(11):1281-1284.

3. Potapov EV, Netuka I, Kaufmann F, Falk V, Mehra MR. Strategy for surgical correction and mitigation of outflow graft twist with a centrifugal-flow left ventricular assist system. J Heart Lung Transplant. 2018 May;37(5):670-673.

4. Schmitto JD, Hanke JS, Rojas SV, Avsar M, Haverich A. First implantation in man of a new magnetically levitated left ventricular assist device(HeartMate III). J Heart Lung Transplant. 2015;34(6):858860.

Figure legends:

Figure 1A,1B:Computed tomography showed an outflow graft twist(white arrow)

Figure 1C:The graft angiography showed a graft twisting(white arrow)

Figure 1D,1E:A pull back pressure measurement showed a significant gradient before(white arrow) and after(white arrow head) the twisting

Figure 2A: Subcostal approach. A Rultruck retractor was useful to lift the rib and expose the surgical field.

Figure 2B: Graft twisting.

Figure 2C:The graft is being untwisted with a curved-up Rongeur forceps.

Figure 2D:The graft was untwisted and fixed. 

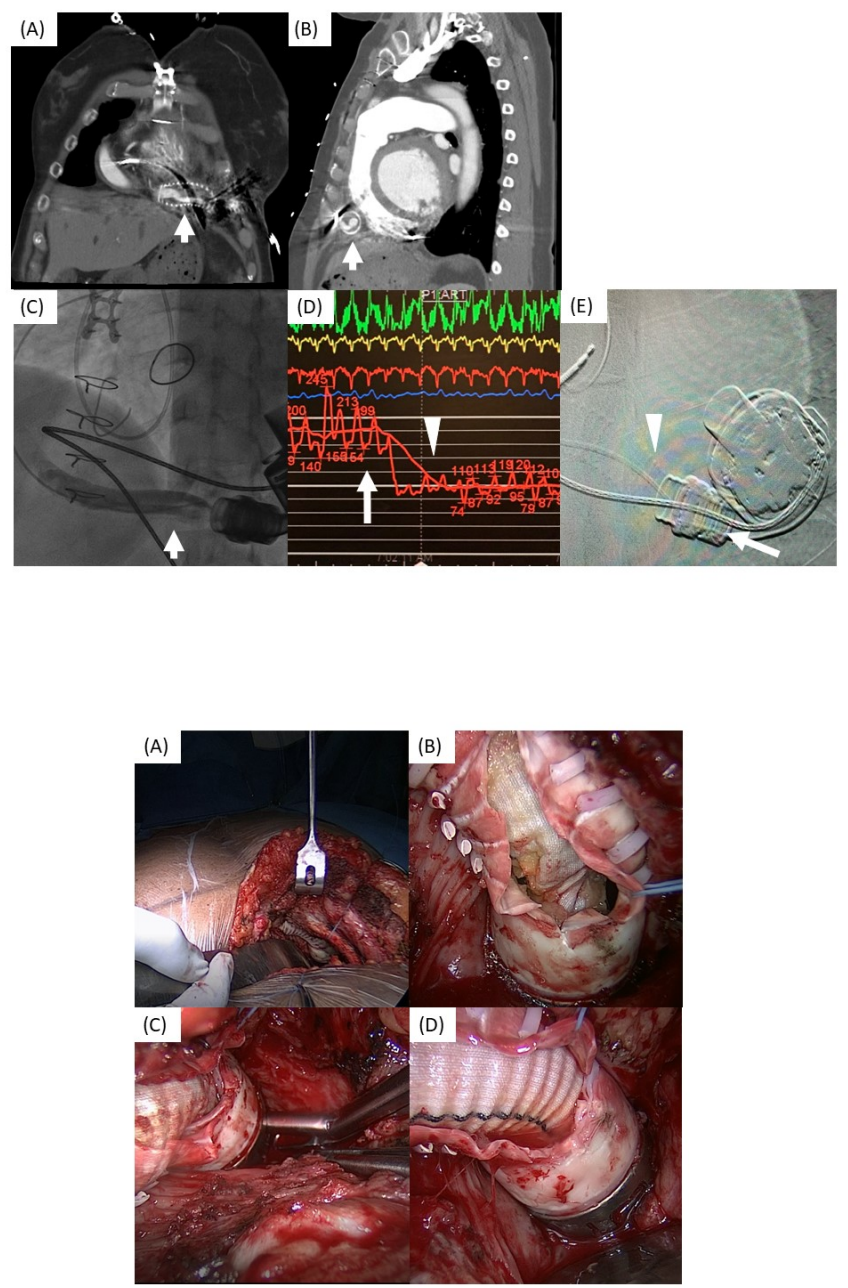